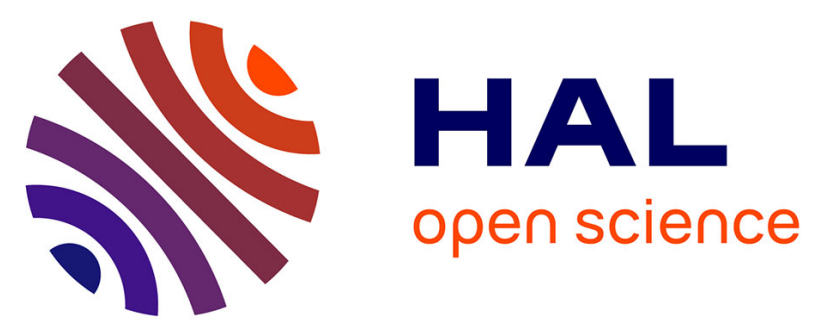

\title{
ASICS: an $R$ package for a whole analysis workflow of 1D 1H NMR spectra
}

\author{
Gaëlle Lefort, Laurence Liaubet, Cécile Canlet, Patrick Tardivel, \\ Marie-Christine Pere, Hélène Quesnel, Alain Paris, Nathalie Iannuccelli, \\ Nathalie Vialaneix, Rémi Servien
}

\section{To cite this version:}

Gaëlle Lefort, Laurence Liaubet, Cécile Canlet, Patrick Tardivel, Marie-Christine Pere, et al.. ASICS: an R package for a whole analysis workflow of 1D 1H NMR spectra. Bioinformatics, 2019, 35 (21), pp.4356-4363. 10.1093/bioinformatics/btz248 . hal-02626125

\section{HAL Id: hal-02626125 \\ https://hal.inrae.fr/hal-02626125}

Submitted on 26 May 2020

HAL is a multi-disciplinary open access archive for the deposit and dissemination of scientific research documents, whether they are published or not. The documents may come from teaching and research institutions in France or abroad, or from public or private research centers.
L'archive ouverte pluridisciplinaire HAL, est destinée au dépôt et à la diffusion de documents scientifiques de niveau recherche, publiés ou non, émanant des établissements d'enseignement et de recherche français ou étrangers, des laboratoires publics ou privés. 
Systems biology

\title{
ASICS: an $\mathrm{R}$ package for a whole analysis workflow of 1D ${ }^{1} \mathrm{H}$ NMR spectra
}

\author{
Gaëlle Lefort ${ }^{1,2, *}$, Laurence Liaubet ${ }^{2}$, Cécile Canlet ${ }^{3,4}$, Patrick Tardivel ${ }^{5}$, \\ Marie-Christine Père ${ }^{6}$, Hélène Quesnel ${ }^{6}$, Alain Paris ${ }^{7}$, Nathalie lannuccelli ${ }^{2}$, \\ Nathalie Vialaneix ${ }^{1,{ }^{\dagger}}$ and Rémi Servien ${ }^{8,}{ }^{\dagger}$
}

${ }^{1}$ MIAT, Université de Toulouse, INRA, Castanet Tolosan, France, ${ }^{2}$ GenPhySE, Université de Toulouse, INRA, ENVT, Castanet Tolosan, France, ${ }^{3}$ Toxalim, Université de Toulouse, INRA, ENVT, INP-Purpan, UPS, 31027 Toulouse, France, ${ }^{4}$ Axiom Platform, MetaToul-MetaboHUB, National Infrastructure for Metabolomics and Fluxomics, 31027 Toulouse, France, ${ }^{5}$ Institute of Mathematics, University of Wroclaw, Poland, ${ }^{6}$ PEGASE, INRA, Agrocampus Ouest, 35590, Saint-Gilles, France, ${ }^{7}$ Unité Molécules de Communication et Adaptation des Microorganismes (MCAM), Muséum national d'Histoire naturelle, CNRS, CP54, 57 rue Cuvier, 75005 Paris, France, and ${ }^{8}$ INTHERES, Université de Toulouse, INRA, ENVT, Toulouse, France.

*To whom correspondence should be addressed. ${ }^{\dagger}$ Equal contributors.

Associate Editor: XXXXXXX

Received on XXXXX; revised on XXXXX; accepted on XXXXX

\section{Abstract}

Motivation: In metabolomics, the detection of new biomarkers from NMR spectra is a promising approach. However, this analysis remains difficult due to the lack of a whole workflow that handles spectra pre-processing, automatic identification and quantification of metabolites and statistical analyses, in a reproducible way.

Results: We present ASICS, an R package that contains a complete workflow to analyse spectra from NMR experiments. It contains an automatic approach to identify and quantify metabolites in a complex mixture spectrum and uses the results of the quantification in untargeted and targeted statistical analyses. ASICS was shown to improve the precision of quantification in comparison to existing methods on two independent datasets. In addition, ASICS successfully recovered most metabolites that were found important to explain a two level condition describing the samples by a manual and expert analysis based on bucketting. It also found new relevant metabolites involved in metabolic pathways related to risk factors associated with the condition.

Availability: ASICS is distributed as an R package, available on Bioconductor.

Contact: gaelle.lefort@inra.fr

Supplementary information: Supplementary data are available at Bioinformatics online.

\section{Introduction}

Metabolomics is the comprehensive characterization of the small molecules involved in metabolic chemical reactions. It is a promising approach in systems biology for phenotype characterization or biomarker discovery, and it has been applied to many different fields such as agriculture, biotechnology, microbiology, environment, nutrition or health. Complementary analytical approaches, such as Nuclear Magnetic Resonance (NMR) or High-Resolution Mass Spectrometry, can be used to obtain metabolic profiles. These technologies allow routine detection of hundreds of metabolites in different biological samples (cell cultures, organs, biofluids...). But, due to their high complexity and to the large amount of generated signals, the analysis of such data remains a major challenge for high-throughput metabolomics.

This article focuses on NMR data, that is a promising tool to detect interesting biomarkers. The most common approach to deal with ${ }^{1} \mathrm{H}$ NMR spectra is to first divide them into intervals called buckets. The areas under the curve are computed for every bucket and every spectrum and these data are given as inputs to statistical methods to provide a lis of buckets of interest (for instance buckets that are significantly different between two conditions). Since buckets are not directly connected to 
metabolites, this approach requires that ${ }^{1} \mathrm{H}$ NMR experts identify the metabolites from the extracted buckets. Not only is this identification step tedious, time consuming, expert dependent and not reproducible but it also leads to a serious loss of information since the identification of metabolites is restricted to the ones that correspond to extracted buckets (Considine et al., 2018).

Some methods have thus been developed to automatically identify metabolites from ${ }^{1} \mathrm{H}$ NMR spectra (MetaboHunter (Tulpan et al., 2011), MIDTool (Filntisi et al., 2017)) and others to automatically quantify the concentration of detected metabolites (Autofit (Weljie et al., 2006), batman (Hao et al., 2012), Bayesil (Ravanbakhsh et al., 2015) and rDolphin (Cañueto et al., 2018)); see Bingol (2018) for a complete review. Recently, Tardivel et al. (2017) defined a new statistical method to automatically identify and quantify metabolites that outperforms the other approaches. However, the approach mainly focuses on the quantification step and needed to be embedded in a complete pre-processing and postprocessing analysis workflow, available through a simple tool. To ou knowledge, such analysis workflows already existed (see a review in Misra (2018)) but they were usually restricted to some steps of the global analysis (post-processing, bucketing or statistical analysis). The only exception seems to be the W4M e-infrastructure (Guitton et al. (2017), available through the Galaxy platform ${ }^{1}$ ), whose automatic identification and quantification step is based on an earlier version of ASICS but the environment only allows one-by-one spectrum analysis. Furthermore, none of the existing workflow is as flexible, easily installed and embedded with other tools than an $\mathrm{R}$ package can be.

The $\mathrm{R}$ package ASICS (Automatic Statistical Identification in Complex Spectra) was designed to fill this gap. The identification an quantification method is partially based on Tardivel et al. (2017) but has been strongly revisited and improved to provide a fine tuning of all the parameters. Changes on the identification step (library distortion) an on the quantification step (model fitting) have also been implemented to improve the results and to reduce the computational cost. In addition the method, that was only available under the form of separate and undocumented scripts, is now properly packaged and documented and the preprocessing of the spectra and post quantification statistical analyses have been implemented and are now part of the pipeline.

\section{Material and methods}

ASICS is an R package available on Bioconductor (Gentleman et al. (2004), http://bioconductor.org/packages/ASICS/) that combines all the steps of the analysis of ${ }^{1} \mathrm{H}$ NMR spectra (library of pure spectra management, preprocessing, quantification, post-quantification statistical analyses). The package also includes functions to directly perform statistical analyses on buckets and diagnosis tools to assess the quality of the quantification. All functionalities of the ASICS package are summarized in Figure 1 and described in the next sections.

\subsection{Preprocessing the complex mixture spectrum}

After the data are imported from raw 1D Bruker spectral data files or other types of files, several preprocessing steps are recommended in order to remove technical biases. Free Induction Decay ou décroissance de l'induction libre

Baseline correction Most of ${ }^{1} \mathrm{H}$ NMR spectra have baseline distortion coming from various sources like instrument instability. These distortions can induce an increase or a decrease in peak intensities and skew the results of quantification. Wang et al. (2013) developed a method to estimate the baseline for a spectrum by classifying each point as a signa

\footnotetext{
1 https://usegalaxy.org/
}

or a noise point and by using a linear interpolation between noise points to construct the baseline. Then, the baseline is subtracted from its spectrum.

Peak alignment Due to $\mathrm{pH}$ or temperature variations between the acquisition of multiple spectra, peak positions of the same metabolite can change between spectra. It is better to align all peaks before analyses, especially if a binning algorithm is used. Vu et al. (2011) developed an algorithm, implemented in the $\mathrm{R}$ package speaq, to carry out this alignment. It is based on continuous wavelet transform to detect peaks and hierarchical clustering to align all spectra on a reference one.

Removal of unwanted regions It is also frequent to exclude a part of the spectra from the analysis. For instance, the part corresponding to water (4.5-5.1 ppm) is of no interest for most biological analyses and thus frequently removed prior to statistical analyses. Urea region (5.5-6.5 ppm) is also frequently excluded in case of urine samples.

Normalisation A normalisation is mandatory before any analysis to make samples comparable. It allows to minimise systematic variations due to differences in sample dilutions. One of the most used methods is the normalisation to a constant sum (Craig et al. (2006)). As a result, the total spectral intensity is the same for each spectrum.

In ASICS, all preprocessing steps are available and the normalisation is the only mandatory one (it is systematically performed when the data are loaded). In the two following steps of the quantification method (preprocessing of the reference library, described in Section 2.2, and quantification itself, described in Section 2.3), all complex mixture spectra are processed individually and independently from each other. The method is thus described for only one complex mixture spectrum (and repeated similarly for all the others).

\subsection{Preprocessing the reference library}

A library of pure metabolite spectra is used as a reference to identify and quantify metabolite concentrations in the (complex mixture) spectra of interest. This library is a set of spectra of pure compounds, that have been acquired independently from samples. Such a reference library is available in ASICS. This library is composed of 190 spectra for which the noise has already been removed (Supplementary Data 1). The spectra acquisition procedure is detailed in Tardivel et al. (2017). In addition, ASICS provides functions to add or remove some spectra from the available reference library or to use another (user provided) reference library.

In addition to removing noise of each library spectrum, preprocessing steps are needed to clean and adapt the library to each spectrum of interest.

Noise thresholding As this is the case for each ${ }^{1} \mathrm{H}$ NMR spectrum, all spectra in library contain noise. All values below a certain threshold, $s_{l}$, (that can be defined by the user; default value is $s_{l}=1$ ), are considered as noise and set to 0 . This allows to select peak positions, a step that is critical for the next selection stage.

First selection step A metabolite can not belong to the complex mixture if at least one peak of its spectrum does not appear in the complex mixture spectrum peaks. Using this simple property, a first selection step is performed. All spectra in the reference library for which the peaks are not included in the peaks of the complex mixture spectrum are removed. This step results in a reference library of $p$ pre-selected reference spectra that are used in the model described in Section 2.3. As technical biases can yield to chemical shifts, a reference spectrum is selected if all its peaks are present in the complex mixture spectrum with an allowed shift of $M$ ppm between the two spectra. In addition, as complex mixture spectra are noisy, peaks under a threshold $s_{m}$ are ignored for this identification step. By default, the maximum allowed shift is $M=0.02 \mathrm{ppm}$ and the 


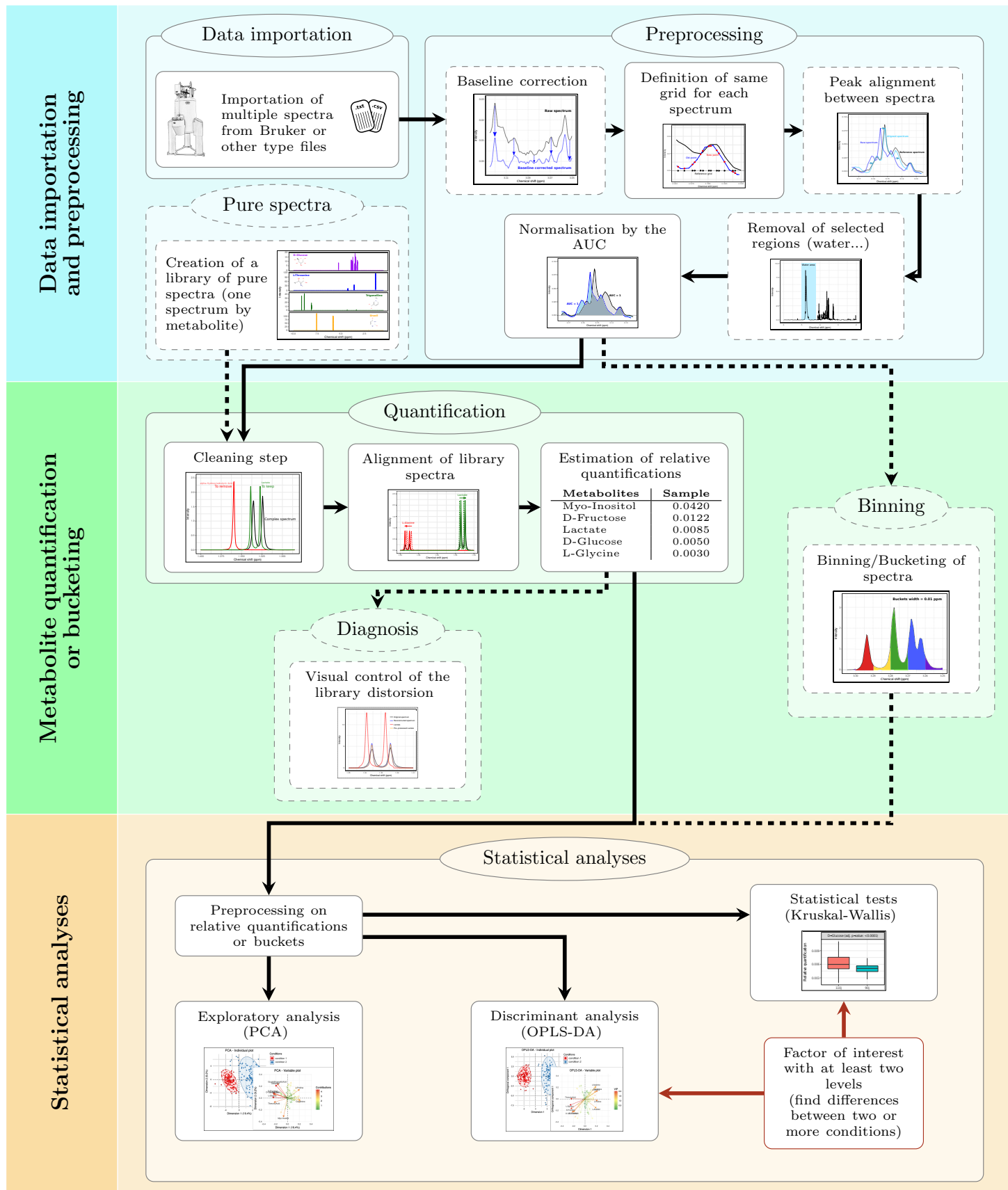

Fig. 1. Schematic representation of ASICS workflow. Bottom box (with brown background): supplementary data (factor corresponding to experimental conditions for the different spectra) are required for this part of the analysis.

threshold is $s_{m}=0.02$. These values have been calibrated on various real datasets with the help of NMR experts. However, both values can be changed by the user, depending on his spectrometer and experimental conditions.

Translation and distortion The alignment algorithm described in Section 2.1 can not be used to align reference spectra with the complex mixture spectrum. The reason is that this method is not adapted to spectra with a low number of peaks as those of the pure metabolite contained in the reference library. Compared to Tardivel et al. (2017), this step is now split into two parts: a first step was added to globally shift the spectrum before a local peak distortion is performed in a second step (Figure 2):
1. First, reference library spectra are aligned with the complex mixture spectrum of interest by maximizing the Fast Fourier Transform crosscorrelation (Wong et al. (2005)). The algorithm that finds the best shift (with a maximum allowed shift equal to $M$ ) is taken from the $\mathrm{R}$ package speaq (Vu et al. (2011))

2. Second, every peak of each library spectrum taken individually is aligned by a local linear regression centered around each peak between the spectrum of interest and the reference library spectrum. To perform local distortions of the chemical shift grid for each peak, ASICS uses the function $\phi(x)=a x(1-x)+x$, where $x \in[0,1]$, corresponds to the rescaled initial grid, $\phi(x) \in[0,1]$ to the newly 
scaled grid and $a \in\left[-\frac{\frac{m}{w_{2}-w_{1}}}{0.5^{2}}, \frac{\frac{m}{w_{2}-w_{1}}}{0.5^{2}}\right] \cap[-1,1]$ is a coefficient of distortion. The definition domain of $a$ is controlled by $m$, the maximum allowed shift (with $m=\frac{M}{5}$ ), and by $\left(w_{1}, w_{2}\right)$ that are the lower and upper bounds of the initial grid, respectively. For each peak, different values of $a$ are tested within this domain and the one that minimizes the residuals of the local linear regression is selected to distort this given peak. This results into a new (distorted) reference library used in the quantification algorithm.

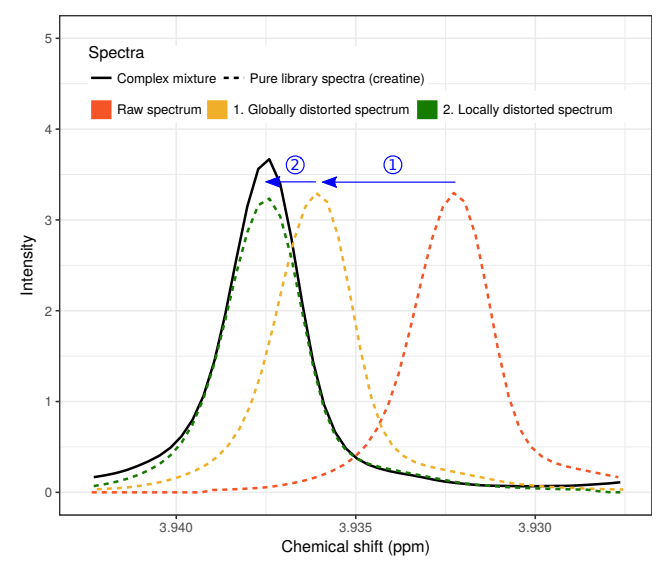

Fig. 2. Two steps distortion procedure for the main peak of the creatine (1) Global translation of the creatine spectrum. (2) Local distortion of one of the creatine peak.

\subsection{Metabolite quantification}

Using the preprocessed complex mixture spectrum and the preprocessed spectra of the reference library, the metabolite identification and quantification in the complex mixture spectrum is performed similarly as in Tardivel et al. (2017). More precisely, the quantification methods does not use the Lasso (that gives biased estimates) anymore but it has been replaced by an faster unpenalized estimation followed by the control of the Family Wise Error Rate (FWER). The complex mixture spectrum is defined as a linear combination of the library reference spectra: $g(t)=$ $\sum_{i=1}^{p} \beta_{i} f_{i}\left(\Phi_{i}(t)\right)+\epsilon(t)$, with $\beta_{i} \geq 0$, where $g$ corresponds to the complex mixture spectrum, $f_{i} \circ \Phi_{i}$ to the $p$ pre-selected preprocessed spectra of the reference library, $\beta=\left(\beta_{1}, \ldots, \beta_{p}\right)$ to the coefficients associated with these spectra (or, equivalently, with the corresponding metabolites) and $\epsilon$ to the noise. The noise is structured so as to take into account both an additive noise, $\epsilon_{2}$, and a multiplicative noise, $\epsilon_{1}$ : $\epsilon=\sqrt{\sum_{1 \leq i \leq p} \beta_{i} f_{i} \circ \Phi_{i}} \epsilon_{1}+\epsilon_{2}$.

A variable selection procedure is implemented to obtain a sparse $\beta$ by controlling the Family Wise Error Rate (FWER) with a risk $\alpha$. Usually, the threshold for rejecting $\mathcal{H}_{0}: \beta_{i}=0$ is the same for every $i$. Here, we used the procedure described in Tardivel (2017) that allows to define metabolite dependent thresholds in order to maximize the test power. More precisely, the custom thresholds $b_{i}$ are computed to minimize the volume of the acceptance region, namely $\arg \min _{\left(b_{i}\right)_{i}} \prod_{i=1}^{p} b_{i}$ subject to $\mathbb{P}^{\mathcal{H}_{0}}\left(\left|\hat{\beta}_{1}\right| \leq b_{1}, \ldots,\left|\hat{\beta}_{p}\right| \leq b_{p}\right)=1-\alpha$, where $\left(\hat{\beta}_{i}\right)_{i=1, \ldots, p}$ are MLE estimates of the previous linear model. The solution of this optimization problem is obtained by simulating a large number of realizations of the random variable $Z \sim \mathcal{N}(0, \Sigma)$, where $\Sigma$ is the estimated variance of the estimates $\left(\hat{\beta}_{i}\right)_{i}$ so as to have $\mathbb{P}^{\mathcal{H}_{0}}\left(\left|\hat{\beta}_{1}\right| \leq\right.$ $\left.b_{1}, \ldots,\left|\hat{\beta}_{p}\right| \leq b_{p}\right)=\mathbb{P}\left(\left|Z_{1}\right| \leq b_{1}, \ldots,\left|Z_{p}\right| \leq b_{p}\right)$, and the thresholds $\left(b_{i}\right)$ are obtained as the $1-\alpha$ quantile of the random variable $\left\{\left|Z_{1}\right|, \ldots,\left|Z_{p}\right|\right\}$, that allows to control the FWER.

Once the metabolites selected, the quantifications $\left(\beta_{i}\right)_{i}$ for those selected metabolites are re-estimated by restricting the previous linear model to this subset in order to limit estimation bias. Finally, the relative quantifications are obtained by dividing $\left(\hat{\beta}_{i}\right)_{i}$ by the respective number of protons of each selected metabolite. In ASICS, pure library preprocessing and quantification are implemented in a unique function that can be run at once for several spectra with a parallel computing backend.

\subsection{Post-quantification statistical analyses}

On quantified metabolites (or on a subset of metabolites that are sufficiently frequently observed in the whole set of complex mixture spectra), the following analyses can be performed:

Quantification assessment To assess the quality of ASICS quantification, a plot with the original complex mixture spectrum, $g(t)$, and the reconstructed spectrum, $\sum_{i=1}^{p} \beta_{i} f_{i}\left(\Phi_{i}(t)\right)$, can be obtained for a given sample (Supplementary Figure $S 1$ ). In addition, one reference spectrum for a given metabolite, and its distorted spectrum, can be superimposed to this plot in order to assess the quality of the metabolite selection for metabolites of interest.

Exploratory analysis To explore results and detect outliers or batch effects, Principal Component Analysis (PCA) can be performed. Individual and variable plots are available to ease the visualisation and interpretation of PCA results (Supplementary Figure S2).

Discriminant analysis When the samples correspond to two experimental conditions, Orthogonal Projections to Latent Structures Discriminant Analysis (OPLS-DA, Trygg and Wold (2002)) can be performed to find the metabolites with the highest discriminant power between these two conditions with a dedicated function based on the implementation available in the ropls package (Thévenot et al., 2015). Prediction error and variable importance in projection (VIP) are computed by a 10 -fold cross-validation procedure, with a stability index for the VIP based on the results of the folds. Individual and variable plots are also available (Supplementary Figure S3).

Statistical tests To find differentially quantified metabolites, statistical tests have also been implemented. Since relative quantifications are usually non normally distributed, Kruskal-Wallis tests are used to find differences between the two (or more) groups, in combination with a correction for multiple testing, as available in the $\mathrm{R}$ function $\mathrm{p}$. adjust. Boxplots showing the differences in metabolite quantification between the conditions can be displayed (Supplementary Figure S4).

\section{Case studies}

\subsection{Plasma metabolome at the end of gestation in piglets}

Genetic selection performed during the last decades has been associated with an increase in perinatal mortality in domestic pig, Sus scrofa (Canario et al., 2006, 2007). One main factor related to neonate survival is the maturation of fetal tissues and organs in late gestation (Voillet et al., 2014; Yao et al., 2017; Voillet et al., 2018; Gondret et al., 2018). In order to explore the development of the metabolic status in late gestation, an experiment was performed on pig fetuses. Metabolomic data were acquired on plasma samples collected on $n=155$ Large White (LW) fetuses at 90 days of gestation and on $n=128$ fetuses at 110 days of gestation (birth is around 114 days; ANR PORCINET project). All ${ }^{1} \mathrm{H}$ NMR spectra were phased and baseline corrected. Glucose, fructose, and lactate were directly quantified by standard methods (they have been 
chosen as indicators of carbohydrate metabolism). More details about the experimental design and data acquisition can be found in Supplementary Section S2.

Similar analyses were performed on buckets and on relative quantifications computed with ASICS to assess the performance of the method. The aim of these analyses was to find metabolites best explaining the differences between the two groups: fetuses at 90 and 110 days of gestation. Lists of metabolites obtained with both approaches were compared as well as the direction of change between groups, based on two OPLS-DA, one on buckets and the other on quantifications obtained with ASICS. VIP thresholds for both OPLS-DA were set to 1.

Metabolites that were quantified were used to make a quantitative assessment of ASICS by comparing the obtained (estimated) quantifications with the dosages. Pearson correlation between quantifications and dosages were computed for every metabolite directly measured by dosage. These correlations were also compared with the correlations obtained for other quantification methods: Autofit, batman, Bayesil and rDolphin. Contrary to ASICS, these methods were too slow or not automated to allow the quantification for the 283 spectra. Therefore, quantifications were performed on a subsample of the original dataset that corresponded to the deciles of the lactate, fructose and glucose dosage to ensure representativity (32 spectra). Computational times were also recorded. For ASICS quantifications, water and urea regions were excluded and the maximum shift, $M$, was set to 0.01 . In order to perform all quantifications with batman in a reasonable time, its library was reduced to the 160 common metabolites between batman and ASICS reference libraries and the number of iterations was set to 10,000

\subsection{Urinary metabolome of Type 2 diabetes mellitus}

In order to test our method on data acquired with another spectrometer than the one on which the pure metabolite library included in ASICS has been obtained, we used the public datasets from Salek et al. (2007). The experiment has been designed to improve the understanding of early stage of type 2 diabetes mellitus (T2DM) development. ${ }^{1} \mathrm{H}$ NMR human metabolome was obtained from 84 healthy volunteers and 50 T2DM patients. Raw 1D Bruker spectral data files were found in the MetaboLights database (Haug et al. (2013); study MTBLS1). In the original study, spectra were normalized by the area under the curve after excluding water (4.24-5.04 ppm), urea (5.04-6.00 ppm) and glucose (3.19-3.99 ppm, 5.21-5.27 ppm) regions. Finally, a bucketing was performed with a $0.04-\mathrm{ppm}$ width. The original study used a combination of PLS-DA and statistical tests ( $t$-test, $F$-test, KruskalWallis test and Kolmogorov-Smirnov test) on buckets (with a manual expert identification) to find differences between the healthy and ill individuals. This dataset allowed us to test the performance of ASICS on a different fluid (urine) in a different species (human).

Contrary to Salek et al. (2007), we kept glucose region for a quantification with ASICS because the glucose spectrum was available in the library. However, regions of water and urea were excluded. The other parameters of the different methods were set to their default values except for ASICS threshold that was set to $s_{m}=0.05$, because we had observed that this dataset was noisier than the previous one. In addition, to control differences that could originate from the analysis method itself, we performed the comparison between the buckets and the ASICS quantifications with the same method, OPLS-DA, as for the study about perinatal survival (VIP thresholds set to 1.2).

\section{Results and discussion}

\subsection{Comparison with biochemical dosages on piglets}

Correlations between quantifications and biochemical dosages of the three metabolites were performed on the 32 selected spectra. We were not able to obtain quantifications with Bayesil because no chemical shift reference (TSP) has been added during spectrum acquisition. Bayesil handles spectrum from raw NMR induction-decay signal and so it requires that spectra are collected with TSP added to the sample (Ravanbakhsh et al., 2015; Beirnaert et al., 2018), TSP is sometimes used as an internal reference in samples for NMR. This procedure is not advised for plasma metabolome, and thus not routinely applied, since TSP binds to plasma proteins (Beckonert et al., 2007).

Table 1 provides the correlations between the quantified target metabolites and their corresponding dosages for the different quantification methods. In addition, the table includes the correlation between one bucket of the target metabolite and the corresponding dosage as a reference value. These results show that ASICS outperforms Autofit, batman and rDolphin for the three metabolites and provides quantification whose correlations are identical to the ones obtained with a direct comparison to the buckets. Results obtained with batman and the library with 160 metabolites are consistent with findings of other studies: the method is not suited for untargeted approaches (Tardivel et al., 2017; Beirnaert et al., 2018). If the quantification with batman is performed including only the three targeted metabolites in the reference library, correlations become similar to the ones obtained by the other methods, but are still lower than those obtained by ASICS with no prior selection of the reference library.

On a practical point of view, ASICS has other interesting features: first, it provides an easy way to handle (complement, replace, manipulate) the reference library whereas batman and rDolphin need that information on each multiplet (chemical shift position, multiplicity...) is specified. A biochemical expertise is thus required for the modification of the reference library in these packages. Autofit is a commercial software that requires the acquisition of a license, which strongly limits its use. Finally, the reference library cannot be modified in Bayesil and this method is only available through a web interface that makes automation of several spectra processing impossible.

In terms of computational times, the preprocessing of the library and the metabolite quantification with ASICS takes about 1'30 min per spectrum and can be launched at once in parallel. A parallel environment is also available for batman but the quantification of a single spectra takes approximately 2 days because of the use of a Bayesian framework that requires extensive MCMC simulations. Computational time needed by rDolphin is approximately the same than for ASICS but paralle implementation is not proposed in the package. Only Autofit has a lower computation time than ASICS (less than one minute) but spectra can only be quantified sequentially (no parallel environment).

A table summarizing capabilities of each method is available in Supplementary Table S3.

\subsection{Differences between gestational ages of fetuses}

For the study about fetuses in late gestation, two outliers were detected on the bucket dataset in a preliminary study (Supplementary Figure S5) and were removed from the analysis (Supplementary Figures S6 and S7).

OPLS-DA was performed on quantified metabolites and on buckets. Both showed the same predicting power: all samples were perfectly separated according to their stages of gestation. For the bucket analysis, VIP values identified 268 buckets on 781 that were found influential to separate the two groups. Based on this list, a manual identification performed by an NMR expert hightlighted 21 metabolites. 
Table 1. Correlation between biochemical dosages of three metabolites and relative quantifications obtained with four methods and the buckets. Bucket for lactate: 1.335; bucket for fructose: 3.995; bucket for glucose: 5.235. Computational time is given for one spectrum.

\begin{tabular}{lccccc}
\hline & Lactate & Fructose & Glucose & $\begin{array}{c}\text { Computational } \\
\text { time }\end{array}$ & $\begin{array}{c}\text { Parallel } \\
\text { environment }\end{array}$ \\
\hline ASICS & 0.93 & 0.95 & 0.90 & $\sim 1$ '30 min & Yes \\
Autofit & 0.52 & 0.74 & 0.75 & $<1 \mathrm{~min}$ & No \\
batman (with 160 metabolites) & 0.46 & 0.56 & 0.22 & $\sim 2$ days & Yes \\
batman (with 3 metabolites) & 0.55 & 0.70 & 0.82 & $\sim 45 \mathrm{~min}$ & Yes \\
rDolphin & 0.82 & Not available & 0.77 & $\sim 1$ '30 min & No \\
Buckets & 0.93 & 0.95 & 0.90 & $2 \mathrm{~s}$ & Yes \\
\hline
\end{tabular}

The same analysis was performed on the ASICS quantifications and allowed to obtain 22 metabolites. The results obtained by ASICS and buckets analysis are detailed in Supplementary Table S4. Nine metabolites were found common to both analyses (Supplementary Figure S8): lactate, creatinine, fructose, glucose, threonine, valine, alanine, proline and leucine. For the metabolites which were not identified by both approaches, we observed five cases:

- metabolites only identified on buckets because the pure spectra was not present in the ASICS reference library: the 3-methyl-2-oxovaleric acid and the lipids;

- metabolites that were identified by ASICS but not selected as influential whereas the buckets corresponding to their peaks were: the betaine and the glutamic acid. Those metabolites indeed exhibited differences between the two groups (that were found significant by a Kruskal-Wallis test) but OPLS-DA did not select them as the most influential. This might be due to the fact that a fixed threshold of VIP equal to 1 is not be equivalent in the two approaches (ASICS quantification and direct bucket analysis). Also, dimension reduction performed with the quantification could have led to a modification of the correlation structure that determines which variables are the most influential in the OPLS-DA model;

- metabolites with low intensity peaks because ASICS was not able to identify and quantify smaller quantities: citrate, tyrosine, lysine, creatine and isoleucine;

- metabolites that were found by ASICS and not by the bucket analysis but for which all peaks corresponded to buckets that were found influential in the bucket analysis: the glycine and the guanidinoacetic acid. For this case, it is very likely that the non identification of these metabolites comes from an expertise bias (peaks are confused with glucose and fructose thus the expert does not identify it);

- metabolites for which no clear conclusion could be driven on their presence without expert knowledge in NMR or biology or the help of other technologies like 2D NMR spectrometry. For ASICS analysis these metabolites correspond to metabolites whose spectra have peaks only in the region with a high density of peaks ( 3.5 to $4.2 \mathrm{ppm}$ threonic acid, xylitol, sorbitol, galactitol, glucolic acid and arabitol), with a low concentration ( $\mathrm{N}$-acetylglycine, acetamidomethylcysteine, arginine and isovaleric acid) or with peaks confused with glucose peaks (glucose-6-phosphate).

The metabolites found by ASICS are consistent with known biological processes of late gestation in pig, especially with the fetal two-fold increase of weight during the last three weeks. It is expected to find up-regulation of the protein synthesis in late gestation, which is illustrated by the increase of amino acid abundances (alanine, proline, threonine, arginine, leucine, valine) just before birth. Also, functiona analysis performed with IPA (see Supplementary Figure S9) highlighted 13 metabolites (among the 22 identified by ASICS) involved in common metabolic pathways directly related to late stage gestation (survival or organism, metabolism of protein, conversion of lipid). Among these metabolites, 6 (guanidinoacetic acid, sorbitol, glucose-6-phosphate, glycine, gluconic acid and arginine) were identified only by ASICS and not with the bucket approach. In this study, the only weakness of ASICS is thus a tendency to miss low concentrated metabolites, especially if those have peaks only in the region with a high density of peaks.

\subsection{Differences for T2DM patients}

Results for the T2DM study are provided in Supplementary Table S5 and in Supplementary Figure S10. The same conclusions than in Section 4.2 can be driven: some metabolites were extracted by both analyses (creatinine, betaine, hippuric acid, guanidinoacetic acid, alanine, glucose, indoxylsulfate, acetoacetate and trigonelline), some did not have a pure spectra available in the library (phenylacetylglycine and 2PY) and the ASICS algorithm had difficulties to identify metabolites with low concentrations (3-hydroxybutyrate, isoleucine, 2-oxoisovalerate, fumaric acid and butyrate) or with only one proton (allantoin).

In addition, results were compared with those previously obtained by Salek et al. (2007) with the same NMR data and with those of an independent experiment realized on urine samples (among other samples) from T2DM patients with another non targeted metabolomic technology Yousri et al. (2015) (results also given in Supplementary Table S5). Those comparisons highlighted the relevance of ASICS quantification that showed results consistent with previous studies and prior knowledge on Type 2 diabete: some of the metabolites were extracted by ASICS and by bucket quantification, like alanine or acetoacetate (Supplementary Table S5), and have also been identified in Salek et al. (2007). We were also able to extract other metabolites, like the glucose (D-Glucose), the guanidinoacetic acid or the glycerol, that were not previously described because the glucose region was excluded from the study in Salek $e t$ al. (2007). The glycerol was identified both by ASICS and by Salek et al. (2007) in experiments on rats and mice (the glucose region was only excluded in the human dataset and not in the rat and mouse datasets) In all experiments, the glycerol increased in diabetics, which might reflect changes in fatty acids metabolism. With ASICS, the creatinine and its precursor, the guanidinoacetic acid (both also found with buckets), were directly quantified in urine and only the creatinine was previously described in (Salek et al., 2007; Yousri et al., 2015) as down regulated in T2DM. Both these metabolites reflect possible impairment of the renal function in diabetics.

In addition, three metabolites (acetoacetate, acetone and 3hydroxybutyrate) reflected the presence of ketone bodies in urine when complications for diabete are likely to occur (Misra and Oliver, 2015). 3-hydroxybutyrate and acetoacetate are detected by Salek et al. (2007) and Yousri et al. (2015) together with buckets and ASICS. Acetone is only identified as discriminant by ASICS allowing the possibility to reflect the risk of acidocetose in diabetics. Another metabolite rarely identified in T2DM, arabitol (L-Arabitol), was quantified as decreasing only with ASICS and firstly described by Yousri et al. (2015) in urine 
of patients. Together with glucose-6-phosphate, also only identified with ASICS, these metabolites reflect the pentose pathway activity in diabetics. Metabolites associated to this pathway were also previously identified in urine as strongly associated with T2DM development in a diabetic rat model (Sun et al., 2014). Finally, only ASICS allowed the identification of GABA ( $\gamma$-aminobutyric acid), a neuromediator recently identified to be increased in T2DM and related to a lower cognitive functioning observed in some diabetic patients (Van Bussel et al., 2016).

In conclusion, not only was ASICS able to automatically recover the main findings of the bucket and expert analysis, it was also able to extract a number of metabolites that are relevant and confirmed by other independent studies but not found by the bucket and expert analysis (glycerol, guanidinoacetic acid, acetone, arabitol, glucose-6-phosphate and GABA). This untargeted approach allowed to highlight several metabolic pathways linked to Type 2 Diabete Mellitus, as illustrated in Supplementary Figure S11.

\section{Conclusion}

This article presents an $\mathrm{R}$ package, ASICS, integrating a complete analysis workflow of ${ }^{1} \mathrm{H}$ NMR spectra. This pipeline integrates an automatic metabolite identification and quantification method based on a reference library of pure metabolite spectra. ASICS showed better quantification results than existing methods and allowed to perform a complete and reproducible study on several hundreds spectra in only a few hours. Its use on two real world datasets exhibited similar results than the standard analysis on buckets followed by expert manual identification but also allowed to provide new information. For both studies, new metabolites, not extracted by expert identification, were found by ASICS, some of them confirmed by previous and independent studies. Obviously, as is the case for other omics data, in coming to a conclusion on whether the metabolites were really present in samples, a validation would be necessary.

Finally, ASICS still has some limitations: the algorithm had difficulties to identify metabolites with low concentrations or with their peaks all located in a region with a high density of peaks. Future work will tackle this aspect, by trying to couple the information from the whole set of spectra to improve the individual quantification.

\section{Funding}

This project received financial support from French National Agency of Research (PORCINET grant ANR-09-GENM005). The PhD fellowship of Gaelle Lefort is supported by the Digital Agriculture Convergence Lab (\#DigitAg, http://www.hdigitag.fr/, ANR-16-CONV-0004), by the INRA Mathematics and Computer Science Division, by the INRA Animal Genetics Division and by the INRA Animal Health Divison.

\section{References}

Beckonert, O. et al. (2007). Metabolic profiling, metabolomic and metabonomic procedures for NMR spectroscopy of urine, plasma, serum and tissue extracts. Nature Protocols, 2(11), 2692-2703.

Beirnaert, C. et al. (2018). speaq 2.0: a complete workflow for high-throughput 1D NMR spectra processing and quantification. PLoS Computational Biology, 14(3), e1006018

Bingol, K. (2018). Recent advances in targeted and untargeted metabolomics by $\mathrm{nmr}$ and $\mathrm{ms} / \mathrm{nmr}$ methods. High-Throughput, 7(2).

Cañueto, D. et al. (2018). rDolphin: a GUI R package for proficient automatic profiling of 1D 1H-NMR spectra of study datasets. Metabolomics, 14(3), 24

Canario, L. et al. (2006). Between-breed variability of stillbirth and its relationship with sow and piglet characteristics. Journal of Animal Science, 12(84), 3185-96. Canario, L. et al. (2007). Estimation of genetic trends from 1977 to 1998 of body composition and physiological state of Large White pigs at birth. Animal, 10(1), $1409-13$.
Considine, E. et al. (2018). Critical review of reporting of the data analysis step in metabolomics. Metabolomics, 14(1), 7.

Craig, A. et al. (2006). Scaling and normalization effects in NMR spectroscopic metabonomic data sets. Analytical Chemistry, 78(7), 2262-2267.

Filntisi, A. et al. (2017). Automated metabolite identification from biological fluid 1H NMR spectra. Metabolomics, 13(12), 146.

Gentleman, R. et al. (2004). Bioconductor: open software development for computational biology and bioinformatics. Genome Biology, 5(10), R80.

Gondret, F. et al. (2018). Proteomic analysis of adipose tissue during the last weeks of gestation in pure and crossbred Large White or Meishan fetuses gestated by sows of either breed. Journal of Animal Science and Biotechnology, 9(1), 28.

Guitton, Y. et al. (2017). Create, run, share, publish, and reference your LC-MS, FIA-MS, GC-MS, and NMR data analysis workflows with the Workflow4Metabolomics 3.0 Galaxy online infrastructure for metabolomics. The International Journal of Biochemistry \& Cell Biology, 93, 89-101.

Hao, J. et al. (2012). BATMAN - an R package for the automated quantification of metabolites from nuclear magnetic resonance spectra using a Bayesian model. Bioinformatics, 28(15), 2088-2090.

Haug, K. et al. (2013). MetaboLights - an open-access general-purpose repository for metabolomics studies and associated meta-data. Nucleic Acids Research, 41(D1), D781-D786.

Misra, B. B. (2018). New tools and resources in metabolomics: 2016-2017. Electrophoresis, 39(7), 909-923.

Misra, S. and Oliver, N. (2015). Utility of ketone measurement in the prevention, diagnosis and management of diabetic ketoacidosis. Diabetic Medicine, 32(1), $14-23$.

Ravanbakhsh, S. et al. (2015). Accurate, fully-automated NMR spectral profiling for metabolomics. PLOS ONE, 10(5), e0124219.

Salek, R. et al. (2007). A metabolomic comparison of urinary changes in type 2 diabetes in mouse, rat, and human. Physiological Genomics, 29(2), 99-108.

Sun, H. et al. (2014). Metabolomic analysis of diet-induced type 2 diabetes using UPLC/MS integrated with pattern recognition approach. PLOS ONE, 9(3), e93384.

Tardivel, P. (2017). Représentation parcimonieuse et procédures de tests multiples : application à la métabolomique. Ph.D. thesis, Universié Toulouse 3 Paul Sabatier.

Tardivel, P. et al. (2017). ASICS: an automatic method for identification and quantification of metabolites in complex 1D 1H NMR spectra. Metabolomics, 13(10), 109.

Thévenot, E. A. et al. (2015). Analysis of the human adult urinary metabolome variations with age, body mass index, and gender by implementing a comprehensive workflow for univariate and OPLS statistical analyses. Journal of Proteome Research, 14(8), 3322-3335.

Trygg, J. and Wold, S. (2002). Orthogonal projections to latent structures (O-PLS) Journal of Chemometrics, 16(3), 119-128.

Tulpan, D. et al. (2011). MetaboHunter: an automatic approach for identification of metabolites from $1 \mathrm{H}$-NMR spectra of complex mixtures. BMC Bioinformatics, 12(1), 400 .

Van Bussel, F. C. et al. (2016). Increased GABA concentrations in type 2 diabetes mellitus are related to lower cognitive functioning. Medicine, $\mathbf{9 5}(36)$.

Voillet, V. et al. (2014). Muscle transcriptomic investigation of late fetal development identifies candidate genes for piglet maturity. BMC Genomics, $\mathbf{1 5}$, 797.

Voillet, V. et al. (2018). Integrated analysis of proteomic and transcriptomic data highlights late fetal muscle maturation process. Molecular \& Cellula Proteomics, 17(4), 672-693.

$\mathrm{Vu}$, T. et al. (2011). An integrated workflow for robust alignment and simplified quantitative analysis of NMR spectrometry data. BMC Bioinformatics, 12(1), 405 .

Wang, K. et al. (2013). Distribution-based classification method for baseline correction of metabolomic 1D proton nuclear magnetic resonance spectra. Analytical Chemistry, 85(2), 1231-1239.

Weljie, A. et al. (2006). Targeted profiling: quantitative analysis of $1 \mathrm{H} \mathrm{NMR}$ metabolomics data. Analytical Chemistry, 78(13), 4430-4442.

Wong, J. et al. (2005). Application of fast Fourier transform cross-correlation for the alignment of large chromatographic and spectral datasets. Analytical Chemistry, 77(17), 5655-5661.

Yao, Y. et al. (2017). Comparing the intestinal transcriptome of Meishan and Large White piglets during late fetal development reveals genes involved in glucose and lipid metabolism and immunity as valuable clues of intestinal maturity. $B M C$ Genomics, 18(1), 647

Yousri, N. A. et al. (2015). A systems view of type 2 diabetes-associated metabolic perturbations in saliva, blood and urine at different timescales of glycaemic control. Diabetologia, 58(8), 1855-1867. 